\title{
Correction to: CDSnake: Snakemake pipeline for retrieval of annotated OTUs from paired-end reads using CD-HIT utilities
}

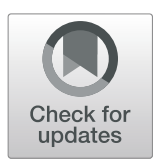

Yulia Kondratenko ${ }^{1 *}$, Anton Korobeynikov ${ }^{1,2}$ and Alla Lapidus ${ }^{1 *}$

\author{
The original article can be found \\ online at https://doi.org/10.1186/ \\ s12859-020-03591-6. \\ * Correspondence: jdkondratenko@ \\ gmail.com; piterlabs@gmail.com \\ From Bioinformatics: from \\ Algorithms to Applications 2019 \\ (BiATA 2019) conference Saint \\ Petersburg, Russia. 20-22 June 2019 \\ ${ }^{1}$ Center for Algorithmic \\ Biotechnology, Institute for \\ Translational Biomedicine, St. \\ Petersburg State University, St. \\ Petersburg, Russia 199004 \\ Full list of author information is \\ available at the end of the article
}

\author{
Correction to: BMC Bioinformatics 21, 303 (2020) \\ https://doi.org/10.1186/s12859-020-03591-6
}

Following publication of the original article [1], the authors would like to correct the Funding note section:

The sentence currently reads: Scientific research performed at the Computing Center of Research park of St. Petersburg State University.

The sentence should read: Scientific research performed at the Computing Center of Research park of St. Petersburg State University. Development of the pipeline and publication of this supplement was funded by Russian Science Foundation grant \#19-16-00049. Author details
'Center for Algorithmic Biotechnology, Institute for Translational Biomedicine, St. Petersburg State University, St.
Petersburg, Russia 199004. ${ }^{2}$ Department of Statistical Modelling, St. Petersburg State University, St. Petersburg, Russia
198515.

Published online: 19 August 2020

\section{Reference}

1. Kondratenko, et al. CDSnake: Snakemake pipeline for retrieval of annotated OTUs from paired-end reads using CD-HIT utilities. BMC Bioinformatics. 2020;21(Suppl 12):303. https://doi.org/10.1186/s12859-020-03591-6. 